International Mathematical Forum, Vol. 8, 2013, no. 14, 653 - 658 HIKARI Ltd, www.m-hikari.com

\title{
On the Extension Problem and the Nil Groups of Rings of Finite Global Dimension
}

\author{
O. S. Olusa \\ Department of Mathematics \\ Adekunle Ajasin University \\ Akungba, Nigeria \\ olusafem@hotmail.co.uk \\ S. A. Ilori \\ Department of Mathematics \\ University of Ibadan \\ Ibadan, Nigeria \\ ailori@mailandnews.com \\ M. EniOluwafe \\ Department of Mathematics \\ University of Ibadan \\ Ibadan, Nigeria \\ michael.enioluwafe@gmail.com
}

Copyright (c) 2013 O. S. Olusa et al. This is an open access article distributed under the Creative Commons Attribution License, which permits unrestricted use, distribution, and reproduction in any medium, provided the original work is properly cited.

\begin{abstract}
A vanishing result is obtained in respect of nil groups of rings of finite global dimension. Also a connection is established with the extension problem.
\end{abstract}

Mathematics Subject Classification: 55N15, 55U05

Keywords: Nil group, global dimension, extension problem 


\section{Introduction}

Let $\mathrm{C}$ be an admissible subcategory of an abelian category. We are interested in the category $\operatorname{Nil}(\mathrm{C})$ whose objects are the pairs $(M, \nu)$, where $M \in \mathrm{C}$ and $\nu \in \operatorname{End}_{\mathrm{C}}(M)$ is nilpotent. The nil groups have geometrical significance as they occur as obstructions to geometrical problems ( [4],[7]). The Nil group vanishes for any abelian category (Proposition 6.1 on page 653 of [2]), and it is an interesting problem to determine under what conditions will the Nil group vanish for a nonabelian category [11]. Some known vanishing results in respect of nil groups for rings are those for the group $\operatorname{ring} \mathbb{Z}[G]$ where $G$ is a finite group of square - free order [5], regular rings ([7],[11]), quasi-regular rings [7], the cyclic group $C_{n}$ of finite order $n \geq 2$ and the finite group of finite type $G \cong F \rtimes \mathbb{Z}$ where $F$ is a finite subgroup of $G$ [8]. This paper gives a solution of the above stated problem in respect of the nonabelian category of rings. We obtain that

$$
N K_{i}(R)=0 \forall i
$$

for a ring $R$ of finite global dimension.

Let $M^{\prime}, M^{\prime \prime}$ be $R$ - modules. The question is asked as regards the $R$ modules $M$ such that $M^{\prime}$ is a submodule of $M$ and $M^{\prime \prime}$ be its quotient. Equivalently, this question can be posed as follows: which are the $R$ - modules $M$ such that the sequence

$$
0 \longrightarrow M^{\prime} \longrightarrow M \longrightarrow M^{\prime \prime} \longrightarrow 0
$$

be exact? The classification of such $R$ - modules $M$ constitute what is known as the extension problem [10]. In this paper, we establish a result that relates the extension problem to rings of finite global dimension. Thus giving us a supply of rings of finite global dimension.

\section{Extensions, Global Dimensions and Nil Groups}

Definition 2.1 An extension of an $R$ - module $M^{\prime}$ by $M^{\prime \prime}$ is a short exact sequence $E: 0 \longrightarrow M^{\prime} \longrightarrow M \longrightarrow M^{\prime \prime} \longrightarrow 0$.

Definition $2.2 \quad$ (i) Let $R$ be a ring and $E$ an $R$ - module. $E$ is said to have homological dimension (denoted $h d_{R} E$ ) $\leq n$ if $\exists$ an exact sequence

$$
0 \longrightarrow P_{n} \stackrel{\partial_{n}}{\longrightarrow} P_{n-1} \stackrel{\partial_{n-1}}{\longrightarrow} \cdots \longrightarrow P_{0} \longrightarrow E \longrightarrow 0
$$

where each $P_{i}$ is projective (i.e. a sum of free $R$ - modules).

(ii) The global dimension of $R$ denoted by gl.dim $R$ is defined as gl.dimR $=\sup h d_{R} E \quad \forall \quad R$-modules $E$ 
On extension problem and nil groups

(iii) The finitistic global dimension of $R$ denoted by f.gl.dim $R$ is defined as f.gl.dim $R=\sup h d_{R} E \quad \forall \quad R$-modules $E \ni h d_{R} E<\infty$.

(iv) Let $G$ be a group, $R[G]$ its corresponding group ring and $\epsilon: R[t] \longrightarrow R$ the augmentation map. The $i$ - th nil group of $R$ is defined as

$$
N K_{i}(R)=\operatorname{ker}\left(K_{i}(R[t]) \stackrel{\epsilon_{*}}{\longrightarrow} K_{i}(R)\right)
$$

(v) Let $G$ be a finite group, $\alpha: \mathbb{Z} \longrightarrow$ Aut $(G)$ a homomorphism and $V=$ $G \rtimes_{\alpha} \mathbb{Z}$ the semidirect product. The nil groups of $\mathbb{Z}[V]$ which takes into account the automorphism $\alpha$, are called twisted nil groups and are denoted by $N K_{i}^{\alpha}(\mathbb{Z}[G])$ i.e.

$$
N K_{i}^{\alpha}(R)=\operatorname{ker}\left(K_{i}\left(R_{\alpha}[t]\right) \stackrel{\epsilon_{*}}{\longrightarrow} K_{i}(R)\right)
$$

(vi) Let $\tau$ be the category of triples $\boldsymbol{R}=\left(R ; B_{0}, B_{1}\right)$, where $B_{i}, i=0,1$ are two bimodules. A morphism in $\tau$ is a triple

$$
\left(\phi, f_{0}, f_{1}\right):\left(R ; B_{0}, B_{1}\right) \longrightarrow\left(S, C_{0}, C_{1}\right)
$$

where $\phi: R \longrightarrow S$ is a ring homomorphism and $f_{i}: B_{i} \otimes_{R} S \longrightarrow C_{i}, i=$ 0,1 , are $R-S$ - bimodule homomorphisms. Waldhausen nil groups are functors from the category $\tau$ to abelian groups. For an object $\boldsymbol{R}$ in $\tau$, we first define an exact category $\operatorname{Nil}(\boldsymbol{R})$ with objects quadruples $(P, Q, ; p, q)$, where $P$ and $Q$ are finitely generated projective right $R$ - modules and

$$
p: P \longrightarrow Q \otimes B_{0}, q: Q \longrightarrow P \otimes B_{1}
$$

is a pair of $R$ - module homomorphisms such that the compositions

$$
\begin{aligned}
& P \stackrel{p}{\longrightarrow} Q \otimes B_{0} \stackrel{q \otimes 1}{\longrightarrow} P \otimes B_{1} \otimes B_{0} \cdots \\
& Q \stackrel{q}{\longrightarrow} P \otimes B_{1} \stackrel{p \otimes 1}{\longrightarrow} P \otimes B_{0} \otimes B_{1} \cdots
\end{aligned}
$$

are zero after finitely many steps. Morphisms are homomorphisms on the modules that are compatible with the maps. There is a forgetful functor $\phi: N i l(\boldsymbol{R}) \longrightarrow \mathcal{P}_{\mathcal{R}} \times \mathcal{P}_{\mathcal{S}}$, where $\mathcal{P}_{\mathcal{R}}$ is finitely generated projective right $R$ - modules. Then the Waldhausen Nil-groups ([7], [9] and [11]) $\widetilde{N i l}_{i}^{W} \boldsymbol{R}$ are defined as

$$
\left.\widetilde{N i l}_{i}^{W} \boldsymbol{R}=\operatorname{ker}\left(K_{i}(\operatorname{Nil}(\boldsymbol{R})) \stackrel{\phi_{i}}{\longrightarrow} K_{i}\left(\mathcal{P}_{\mathcal{R}} \times \mathcal{P}_{\mathcal{R}}\right)\right), \text { for }\right\rangle \in \mathbb{Z} .
$$


Remark 2.3 There is a natural isomorphism between the NK-groups and the Waldhausen Nil-groups

$$
N K_{i}(R) \cong \widetilde{N i l}_{i-1}^{W}(R), \text { for } i \leq 1 .
$$

Thus the vanishing results of Waldhausen Nil - groups can be applied to the NK - groups. ([3])

The group of equivalence classes of extensions of $M^{\prime}$ by $M^{\prime \prime}$ under the Baer sum is denoted by $\operatorname{Ex}\left(M^{\prime \prime}, M^{\prime}\right)$ and it is isomorphic to $\operatorname{Ext}^{1}\left(M^{\prime \prime}, M^{\prime}\right)$ [10].

\section{Nil Groups of Rings of Finite Global Dimen- sion}

It is known that the relationship between the extension bifunctor and the Baer sum is illustrated in the solution to the extension problem ([10]). Then by results from $([12])$, we relate rings of finite global dimension to the extension problem. Thus giving a condition for the supply of rings of finite global dimension.

Theorem 3.1 Let $R$ be a ring of finite global dimension. Then $N K_{i}(R)=0 \forall i$.

Proof: Let $R$ be a ring of finite global dimension. Then $h d_{R} E \leq n<\infty$ for any $R$ - module $E$ and exact sequence

$$
0 \longrightarrow P_{n} \stackrel{\partial_{n}}{\longrightarrow} P_{n-1} \cdots \longrightarrow P_{0} \longrightarrow E \longrightarrow 0
$$

where each $P_{i}$ is projective. Let $p$ be a prime ideal of $R$ and $S$ a multiplicatively closed subset of $R$ not containing 0 given by $S=R-\underline{p}$. The ring of fractions $R_{p}=S^{-1} R$ can give an indication on whether $R$ is regular ([1]). Since $R$ is of finite global dimension, it follows that $R_{\underline{p}}$ also has finite global dimension and is regular. Thus $R$ is regular. It is known that every $R$ - module has a projective resolution and since $R$ is of finite global dimension, it means that every $R$ - module is of homological dimension $\leq n(n \in \mathbb{N})$. Now the category of finitely generated projective modules is a full subcategory of the category of $R$ - modules. Therefore $K_{i}(N i l(\mathrm{R})) \longrightarrow K_{i}\left(\operatorname{Nil}\left(\operatorname{Mod}_{R}\right)\right)$ is an isomorphism. Using Corollary 6.3 on p. 654 of [2] and the fact that the groups $N K_{i}$ are isomorphic to the Waldhausen's groups $\widetilde{N i l}_{i-1}^{W}$ for regular rings (see Proof of Proposition 3.8 in [3]), it follows that $N K_{i}(R)=0 \forall i$.

Theorem 3.2 Let $A$ be a ring such that the extensions of any simple module $S$ by $S$ over $A$ splits and consists of exactly only one element. Then $A$ is of finite global dimension. 
Proof: Suppose that $A$ be a ring such that the extensions of any simple $A$ - module $S$ by $S$ over $A$ splits and consists of only one element. Then by Theorem 3.14 on page 15 of [10] we have that $\operatorname{Ext}_{A}^{n}(S, S)=0 \quad \forall n \geq 1$ and all simple $A$ - modules $S$ and consequently $\operatorname{Ext}_{A}^{n}(S, S)=0$ for all simple $A$ modules $S$ and all $n>>$. Then by Theorem 2 of [12], $A$ is of finite global dimension.

\section{References}

[1] M. Auslander, and D.A. Bauchsbaum, Homological dimension in local rings, Transactions of American Mathematical Society, 85 (1957), 390 405.

[2] H. Bass, Algebraic K - theory, Benjamin, New York, 1968.

[3] F.X. Connolly and S. Prassidis, On the exponent of the $N K_{0}$ - group of virtually infinite cyclic groups, Canadian Mathematical Bulletin, 45 (2002), 180 - 195.

[4] J.F. Davis, Q. Khan and A. Ranicki, Algebraic $K$ - theory over the infinite dihedral group: an algebraic approach, Algebraic $\& 6$ Geometric Topology, 11 (2011), 2391 - 2436.

[5] D. Harmon, $N K_{1}$ of finite group, Proceedings of the American Mathematical Society, 100 (1987), 229 - 231.

[6] D. Juan-Pineda, On higher nil groups of group rings, Homology, Homotopy and Applications, 9 (2007), 95 - 100.

[7] D. Juan-Pineda and S. Prassidis, On the nil groups of Waldhausen nils, Topology and its Applications, 146-149 (2005), 489 - 499.

[8] D. Juan-Pineda and R. Ramos, On the vanishing of twisted nil groups, Journal of $K$ - Theory, 3 (2009), 153 - 163.

[9] J. Lafont and I.J. Ortiz, Splitting formulas for certain Waldhausen nil - groups, Journal of the London Mathematical Society, 79 (2009), 309 322.

[10] E. Lluis-Puebla, Higher $K$ - Theory: an overview IX. Introduction to Algebraic $K$ - Theory. Proceedings of the first seminar on algebraic $K$ - theory at the Universidad Nacional Autonoma de Mexico in 1985, SpringerVerlag, Berlin, 1992. 
[11] H.J. Munkholm and S. Prassidis, On the vanishing of certain K-Theory Nil-groups, Proceedings of Algebraic Topology Conference, Bacelona (1998).

[12] M. Saorin, On semiprimary rings of finite global dimension, Communications in Algebra, 33 (2005), 737 - 743.

\section{Received: January 4, 2013}

